PROCEEDINGS OF THE

AMERICAN MATHEMATICAL SOCIETY

Volume 126, Number 6, June 1998, Pages 1725-1732

S $0002-9939(98) 04520-1$

\title{
MULTIPLICITY OF PERIODIC SOLUTIONS FOR DUFFING EQUATIONS UNDER NONUNIFORM NON-RESONANCE CONDITIONS
}

\author{
CHENGWEN WANG
}

(Communicated by Hal L. Smith)

\begin{abstract}
This paper is devoted to the study of multiple $2 \pi$-periodic solutions for Duffing equations

$$
x^{\prime \prime}+c x^{\prime}+g(t, x)=s(1+h(t))
$$

under the condition of nonuniform non-resonance related to the positive asymptotic behavior of $g(t, x) x^{-1}$ at the first two eigenvalues 0 and 1 of the periodic BVP on $[0,2 \pi]$ for the linear operator $L=-x^{\prime \prime}$, and the condition on the negative asymptotic behavior of $g(t, x)$ at infinity. The techniques we use are degree theory and the upper and lower solution method.
\end{abstract}

\section{INTRODUCTION}

In this paper, we study the multiplicity of periodic solutions for the Duffing differential equation

$$
x^{\prime \prime}+c x^{\prime}+g(t, x)=s(1+h(t)),
$$

where $c$ is a constant, $s$ a parameter, $h:[0,2 \pi] \rightarrow R$ a continuous function and $g:[0,2 \pi] \times R \rightarrow R$ satisfies the Carathéodory condition, i.e., $g(\cdot, x)$ is measurable on $[0,2 \pi]$ for each $x \in R$ and $g(t, \cdot)$ is continuous on $R$ for almost each $t \in[0,2 \pi]$, and moreover, for each $r>0$ there exists a $g_{r} \in L^{1}(0,2 \pi)$ such that $|g(t, x)| \leq g_{r}(t)$ for a.e. $t \in[0,2 \pi]$ and all $x \in[-r, r]$.

A periodic solution $x(t)$ of $(1.1)$ is a map $x:[0,2 \pi] \rightarrow R$, continuously differentiable and satisfying (1.1), such that $x^{\prime}$ is absolutely continuous and $x(0)=$ $x(2 \pi), x^{\prime}(0)=x^{\prime}(2 \pi)$.

There are a lot of papers devoted to the study of the existence of periodic solutions for (1.1); see [1]-[3] and [5]-[7] and the references therein. In [1], for $c=0$ and $g(t, x) \equiv g(x)$ with $g$ continuous, using the Banach fixed-point theorem, and the upper and lower solutions method, Pino et al. proved that (1.1) has at least two periodic solutions, one being strictly positive and unique, and the other strictly negative if $g(x)$ satisfies

$$
\lim _{x \rightarrow-\infty} g(x)=+\infty \quad \text { and } \quad 0<\lim _{x \rightarrow+\infty} g^{\prime}(x)<1
$$

under some roundedness conditions on $s$ and $h(t)$. In [2], for $c=0$ and $g(t, x)$ being continuous and with a singular behavior near the origin, using degree theory, Pino

Received by the editors November 20, 1996.

1991 Mathematics Subject Classification. Primary 34C25, 34 B15. 
et al. proved that (1.1) has at least one positive periodic solution if

$$
\alpha(t) \leq \lim _{x \rightarrow+\infty} \inf g(t, x) x^{-1} \leq \lim _{x \rightarrow+\infty} \sup g(t, x) x^{-1} \leq \beta(t)
$$

with $\alpha(t)$ and $\beta(t)$ between two consecutive eigenvalues of the linear operator $L=$ $-x^{\prime \prime}$ under the non-resonance condition. On the other hand, the author in [6] showed that there exists an $s_{0}$ such that (1.1) has zero, one and two periodic solutions for $s<s_{0},=s_{0}$ and $>s_{0}$ under some boundedness of $\operatorname{esssup}_{t \in[0,2 \pi]} g(t, 0)$ for $h(t) \equiv 0$, using the upper and lower solutions method.

The aim of this paper is to adapt some techniques introduced in [5] by Mawhin et al. and in [3] by Drabek et al., who studied the existence of periodic solutions for $s=0$, to the study of the multiplicity of periodic solutions of (1.1). In [3], the existence of periodic solutions is obtained under the hypothesis of jumping nonlinearity. In [5], the conditions of the main results relate the asymptotic behavior of $g(t, x) x^{-1}$, for $|x|$ sufficiently large, to the first two eigenvalues 0 and 1 of the periodic boundary value problem in $[0,2 \pi]$ for the linear operator $L$. More precisely, it was assumed that

$$
\gamma(t) \leq \lim _{|x| \rightarrow+\infty} \inf g(t, x) x^{-1} \leq \lim _{|x| \rightarrow+\infty} \sup g(t, x) x^{-1} \leq \Gamma(t)
$$

uniformly a.e. in $t \in[0,2 \pi]$, and that $\gamma(t), \Gamma(t)$ satisfy

$$
\gamma(t) \in L^{1}(0,2 \pi), \quad \int_{0}^{2 \pi} \gamma(t) d t \geq 0, \quad \int_{0}^{2 \pi} \gamma^{+}(t) d t>0,
$$

and with the following strict inequality on a subset of $[0,2 \pi]$ of positive measure:

$$
\Gamma(t) \leq 1
$$

where $\gamma^{+}(t)=\max _{t \in[0,2 \pi]}\{\gamma(t), 0\}$.

(1.3) and (1.4) are usually called semi-linear conditions. In some recent papers, some authors have discussed periodic solutions for conservative Duffing equations (in our case, $c=0$ and $g(t, x) \equiv g(x)$ for all $t \in[0,2 \pi)$ ) under the semi-linear condition (1.4). For example, in [8], using the optimal control theory method, Hao and Ma gave the uniqueness of periodic solutions; in [9], Wang and Li obtained the existence of periodic solutions when $g(x)$ crosses the resonance points by "the twist theorem".

In this paper, we will consider the general Duffing equation (1.1). Suppose that $g(t, x)$ is a Carathéodory function with singularity near the origin, i.e., there exist constants $c, c^{\prime}, \delta>0$ and $\nu \geq 1$ such that

$$
c^{\prime} x^{\nu} \leq-g(t, x) \leq c x^{\nu}
$$

for all $x \in(0, \delta)$ and

$$
\lim _{x \rightarrow-\infty} g(t, x)=+\infty
$$

uniformly a.e. in $t \in[0,2 \pi]$, and

$$
\gamma(t) \leq \lim _{x \rightarrow+\infty} \inf g(t, x) x^{-1} \leq \lim _{x \rightarrow+\infty} \sup g(t, x) x^{-1} \leq \Gamma(t)
$$

uniformly a.e. in $t \in[0,2 \pi]$ with $\gamma(t)$ and $\Gamma(t)$ satisfying (1.5) and (1.6) respectively.

We will show that, under our assumptions above, there are at least two periodic solutions for (1.1). Here $\gamma(t)$ and $\Gamma(t)$ satisfying (1.5) and (1.6) implies that the nonlinearity $g(t, x)$ is confined between two consecutive eigenvalues, i.e., (1.1) does 
not cross the resonance points. Our results are mainly motivated by [1], [5] and [6], and are quite in the spirit of [1], [2] and [5].

In order to state our results and for convenience in the proofs, we give the following notations. $C([0,2 \pi])(C(2 \pi))$ denotes the usual Banach space of con-

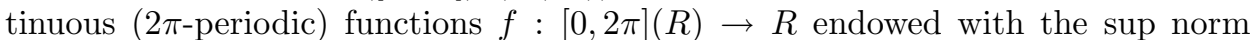
$\|f\|_{0} . C^{k}[0,2 \pi]$ denotes the Banach space of $C^{k}$ functions $f:[0,2 \pi] \rightarrow R$ endowed with the sup norm

$$
\|f\|_{C^{k}}=\sum_{i=0}^{k} \sup _{t \in[0,2 \pi]}\left|f^{(k)}(t)\right| .
$$

$L^{k}(0,2 \pi)$ denotes Lebesgue spaces of real measurable functions whose $k$-th power of the absolute value is Lebesgue integrable. $H^{1}(0,2 \pi)$ is the Sobolev space defined by $H^{1}(0,2 \pi)=\left\{x(t):[0,2 \pi] \rightarrow R \mid x\right.$ is absolutely continuous on $[0,2 \pi]$ and $x^{\prime} \in$ $\left.L^{2}(0,2 \pi)\right\}$, with norm

$$
|x|_{H^{1}}=\left(\int_{0}^{2 \pi} x(t) d t+\int_{0}^{2 \pi} x^{\prime 2}(t) d t\right)^{\frac{1}{2}} .
$$

Finally, as usual, for any $x \in L^{1}(0,2 \pi)$, let

$$
x^{+}(t)=\max _{t \in[0,2 \pi]}\{x(t), 0\} \quad \text { and } \quad x^{-}(t)=\max _{t \in[0,2 \pi]}\{-x(t), 0\} .
$$

\section{EXISTENCE OF NEGATIVE PERIODIC SOLUTIONS}

In this section, we use the upper and lower solutions method introduced by [6] for the Duffing equation

$$
x^{\prime \prime}+c x^{\prime}+f(t, x)=0
$$

to obtain the existence of a negative $2 \pi$-periodic solution for (1.1).

Definition 1. Let $a:[0,2 \pi] \rightarrow R, b:[0,2 \pi] \rightarrow R$ be functions of class $C^{1}$ with absolutely continuous derivatives such that for all $t \in[0,2 \pi]$ one has

$$
a(t) \leq b(t) .
$$

Such functions $a(t)$ and $b(t)$ are called lower and upper solutions, respectively, if they satisfy

$$
\begin{aligned}
& a^{\prime \prime}(t)+c a^{\prime}(t)+f(t, a(t)) \geq 0 \text { for a.e. } t \in I, \\
& a(0)=a(2 \pi), a^{\prime}(0)=a^{\prime}(2 \pi) ;
\end{aligned}
$$

and

$$
\begin{aligned}
& b^{\prime \prime}(t)+c b^{\prime}(t)+f(t, b(t)) \leq 0 \text { for a.e. } t \in I, \\
& b(0)=b(2 \pi), b^{\prime}(0)=b^{\prime}(2 \pi) .
\end{aligned}
$$

The following lemma has been proved in [6] or [7],

Lemma $1([6])$. Assume that there exist a lower solution $a(t)$ and an upper solution $b(t)$ defined by Definition 1 for (2.1). Then (2.1) has at least one solution $x(t)$ such that $a(t) \leq x(t) \leq b(t)$ for all $t \in I$.

Applying Lemma 1, we have

Lemma 2. Suppose that $\|h\|_{0}<1$. Then there exists an $s_{1}>0$ such that (1.1) has a strictly negative $2 \pi$-periodic solution for all $s \geq s_{1}$. 
Proof. Let us first rewrite (1.1) as follows:

$$
x^{\prime \prime}+c x^{\prime}+g(t, x)-s(1+h(t))=0 .
$$

First of all , by (1.8) and (1.6), we have

$$
\lim _{x \rightarrow+\infty} \sup g(t, x)=+\infty .
$$

Otherwise, we will have $\lim _{x \rightarrow+\infty} \sup g(t, x)=$ const and $\lim _{x \rightarrow+\infty} \sup g(t, x) x^{-1} \equiv 0$ uniformly for $t \in[0,2 \pi]$, which contradicts (1.8).

It follows from $\|h\|_{0}<1$ that there exist an $\varepsilon_{0}>0$ such that for all $s>0$

$$
\varepsilon_{0} s \leq s(1+h(t))<2 s .
$$

For such $\varepsilon_{0}$, by (1.7) and (2.6), there exist $s_{1}>0$ and $\bar{x}_{s}<0$ such that $g\left(t, \bar{x}_{s}\right)<\varepsilon_{0} s$ for all $s \geq s_{1}$ and a.e. $t \in I$. Therefore, by (2.7), we have

$$
g\left(t, \bar{x}_{s}\right)<s(1+h(t))
$$

for all $s \geq s_{1}$ and a.e. $t \in I$.

On the other hand, by (2.3) and (1.7), there exists an $\underline{x}_{s}$, with $\underline{x}_{s}<\bar{x}_{s}<0$, such that $g\left(t, \underline{x}_{s}\right)>2 s$ for all $s \geq s_{1}$ and a.e. $t \in I$. Therefore

$$
g\left(t, \underline{x}_{s}\right)>s(1+h(t))
$$

for all $s \geq s_{1}$, and a.e. $t \in I$.

Combining (2.8) and (2.9), we have

$$
g\left(t, \bar{x}_{s}\right)-s(1+h(t))<0<g\left(t, \underline{x}_{s}\right)-s(1+h(t))
$$

for all $s \geq s_{1}$ and a.e. $t \in I$, which implies that $\underline{x}_{s}$ and $\bar{x}_{s}$ are lower and upper solutions of (1.1) respectively. Then, according to Lemma 1, we conclude that (1.1) has at least one $2 \pi$-periodic solution $x_{s}(t)$ for $s \geq s_{1}$ such that $\underline{x}_{s}<x_{s}(t)<\bar{x}_{s}<0$ for all $t \in I$. This completes the proof of our lemma.

\section{Existence of POSITIVE PERIOdiC SOlutions}

This section is devoted to the existence of positive periodic solutions by using degree theory.

Theorem 1. Suppose that $g(t, x)$ is a Carathéodory function with singularity near the origin and satisfies (1.8) with $\Gamma(t)$ and $\gamma(t)$ satisfying (1.5) and (1.6). Then there exist an $h_{0}, 0<h_{0}<1$, and $s_{2}=s_{2}\left(h_{0}\right), s_{2}>0$, such that for all $s \geq s_{2}$ and for all $h \in C(2 \pi)$ with $\|h\|_{0} \leq h_{0}<1$, (1.1) possesses at least one strictly positive $2 \pi$-periodic solution.

In order to prove this theorem, we need the following lemma, introduced by Mawhin et al. in [5].

Lemma 3 ([5]). Suppose that $p \in L^{1}(0,2 \pi)$ satisfies the following conditions:

1. $\int_{0}^{2 \pi} p(t) d t \geq 0, \quad \int_{0}^{2 \pi} p^{+}(t) d t>0$;

2. $p(t) \neq 0$ on a subset of $[0,2 \pi]$ of positive measure; and

3. $p(t) \leq 1$ for a.e. $t \in[0,2 \pi]$, with strict inequality on a subset of positive measure. 
Then the linear periodic boundary value problem

$$
\begin{aligned}
& x^{\prime \prime}+c x^{\prime}+p^{+}(t) x^{+}-p^{-}(t) x^{-}=0, \\
& x(0)=x(2 \pi), x^{\prime}(0)=x^{\prime}(2 \pi),
\end{aligned}
$$

has only the trivial solution.

Remark. This lemma is a combination of Lemma 2 and Lemma 3 in [5].

Proof of Theorem 1. Let us again rewrite (1.1) as

$$
x^{\prime \prime}+c x^{\prime}+g(t, x)-s(1+h(t))=0 .
$$

Let $g_{1}(t, x)=g(t, x)-s(1+h(t))$. Then it follows from (1.8) that there exist an $h_{0}, 0<h_{0}<1$, and $s_{2}=s_{2}\left(h_{0}\right), s_{2}>0$, such that for all $s \geq s_{2}$ and for all $h \in C(2 \pi)$ with $\|h\|_{0} \leq h_{0}<1$ the inequalities

$$
\gamma(t) \leq \lim _{x \rightarrow+\infty} \inf g_{1}(t, x) x^{-1} \leq \lim _{x \rightarrow+\infty} \sup g_{1}(t, x) x^{-1} \leq \Gamma(t)
$$

hold for a.e. $t \in[0,2 \pi]$. Therefore, for a given $\delta=\delta(\gamma, \Gamma) \geq 0$, there exists an $r_{0}>0$ such that for a.e. $t \in[0,2 \pi]$ and all $x>r_{0}$, we have

$$
\gamma(t)-\frac{\delta}{2} \leq x^{-1} g_{1}(t, x) \leq \Gamma(t)+\frac{\delta}{2} .
$$

Let

$$
\widetilde{\gamma}(t, x)= \begin{cases}x^{-1} g_{1}(t, x), & x \geq r_{0}>0 ; \\ r_{0}^{-1} g_{1}\left(t, r_{0}\right), & 0<x<r_{0} ; \\ \gamma(t), & x=0,\end{cases}
$$

and define $\widetilde{g}:[0,2 \pi] \times R \rightarrow R$ by $\widetilde{g}(t, x)=\widetilde{\gamma}(t, x) x$. Then $\widetilde{g}(t, x)$ is also a Carathéodory function, and $\widetilde{\gamma}$ satisfies

$$
\gamma(t)-\frac{\delta}{2} \leq \widetilde{\gamma}(t, x) \leq \Gamma(t)+\frac{\delta}{2}
$$

for a.e. $t \in[0,2 \pi]$ and all $x \in R$.

Let $\widetilde{h}(t, x)=g_{1}(t, x)-\widetilde{g}(t, x)$; then there exists an $\alpha \in L^{1}(0,2 \pi)$, depending on $\gamma, \Gamma$ and $\widetilde{\gamma}$, such that for a.e. $t \in[0,2 \pi]$ and all $x \geq 0$ we have

$$
\widetilde{h}(t, x) \leq \sup _{0 \leq x \leq r_{0}}\left|g_{1}(t, x)-\widetilde{g}(t, x)\right| \leq \alpha(t) .
$$

Hence, (1.1) is equivalent to the periodic boundary value problem

$$
x^{\prime \prime}+c x^{\prime}+\widetilde{\gamma}(T, x) x+\widetilde{h}(t, x)=0 .
$$

Now, let $\operatorname{dom} L=\left\{x \in C^{1}([0,2 \pi]) \mid x \geq 0, x(0)=x(2 \pi), x^{\prime}(0)=x^{\prime}(2 \pi)\right.$ and $x^{\prime}$ is absolutely continuous on $[0,2 \pi]\}$, and

$$
\begin{aligned}
& L: \operatorname{dom} L \subset C^{1}([0,2 \pi]) \rightarrow L^{1}(0,2 \pi): x \rightarrow x^{\prime \prime}(\cdot)+c x^{\prime}(\cdot), \\
& G: C^{1}([0,2 \pi]) \rightarrow L^{1}(0,2 \pi) ; x \rightarrow \widetilde{\gamma}(\cdot, x(\cdot)) x(\cdot), \\
& H: C^{1}([0,2 \pi]) \rightarrow L^{1}(0,2 \pi): c \rightarrow \widetilde{h}(\cdot, x(\cdot)), \\
& A: C^{1}([0,2 \pi]) \rightarrow L^{1}(0,2 \pi): x \rightarrow \widetilde{\gamma}(\cdot, 0) x(\cdot)=\gamma(\cdot) x(\cdot) .
\end{aligned}
$$


It is well known that all the mappings above are well defined, $L$ is a Fredholm operator of index zero, and $G, H$, and $A$ are $L$-compact on bounded subsets of $x$. Therefore, (3.7) is equivalent to the operator equation

$$
L x+G x+H x=0
$$

Consider a homotopy $\widetilde{H}:[0,1] \times C^{2}([0,2 \pi]) \rightarrow L^{1}([0,2 \pi])$ defined by

$$
\widetilde{H}(\lambda, x)=L x+\lambda G x+\lambda H x+(1-\lambda) A x .
$$

In order to apply the well-known Leray-Schauder continuation theorem (see, for example, [4]), all we have to do is to prove that there exist $r_{1}$ and $R_{1}$ with $0<$ $r_{1}<R_{1}$ such that for each $\lambda \in[0,1]$ and each $x(\cdot) \in \operatorname{dom} L$ such that $\widetilde{H}(\lambda, x)=0$ we have $r_{1}<x(t)<R_{1}$ for all $t \in[0,2 \pi]$.

Now, suppose that such $r_{1}$ and $R_{1}$ do not exist. Then we could find sequences $\lambda_{n} \in[0,1], x_{n} \in C^{1}([0,2 \pi]), x_{n}>0$, such that

$$
\widetilde{H}\left(\lambda_{n}, x_{n}\right)=0
$$

and either

$$
\inf _{t \in[0,2 \pi]} x_{n}(t) \rightarrow 0 \quad \text { as } \quad n \rightarrow \infty
$$

or

$$
\sup _{t \in[0,2 \pi]} x_{n}(t) \rightarrow+\infty \quad \text { as } n \rightarrow \infty .
$$

It can be proved similarly to [2] by a slight modification that under the singularity hypothesis on $g(t, x)$ near the origin, which guarantees that $g_{1}(t, x)$ also has a singularity near the origin under the condition of the existence of $s_{0}$ and $h_{0},(3.11)$ and (3.12) are actually equivalent. So we only assume (3.12) in the following, and therefore we can define $\widehat{x}_{n}=x_{n}\left\|x_{n}\right\|_{C^{0}}^{-1}$.

Multiplying (3.10) by $x_{n}(t)$ and integrating by parts, we have

$$
\int_{0}^{2 \pi}\left|\widehat{x}_{n}^{\prime}(t)\right|^{2} d t=\lambda_{n} \int_{0}^{2 \pi} \frac{g_{1}\left(t, x_{n}(t)\right)}{\left\|x_{n}(t)\right\|_{C^{0}}} \widehat{x}_{n}(t) d t+\left(1-\lambda_{n}\right) \int_{0}^{2 \pi} \gamma(t) \frac{\widehat{x}_{n}(t)}{\left\|x_{n}\right\|_{C^{0}}} d t
$$

By (1.8) and (1.6), we have

$$
g_{1}(t, x) \leq m x+k
$$

for positive constants $m$ and $k$, for all $x>0$ and a.e. $t \in[0,2 \pi]$. Then, by (3.13), we have

$$
\int_{0}^{2 \pi}\left|\widehat{x}_{n}^{\prime}(t)\right|^{2} d t \leq \frac{\lambda_{n}}{\left\|x_{n}\right\|_{C^{0}}} \int_{0}^{2 \pi}\left(m x_{n}(t)+k\right) \widehat{x}_{n}(t) d t+\frac{\left(1-\lambda_{n}\right)}{\left\|x_{n}\right\|_{C^{0}}} \int_{0}^{2 \pi} \gamma(t) \widehat{x}_{n}(t) d t
$$

and therefore $\widehat{x}_{n}(t)$ is bounded in $H^{1}(0,2 \pi)$; passing to a subsequence if necessary, we may assume that $\widehat{x}_{n} \rightarrow \widehat{x}$ weakly in $H^{1}(0,2 \pi)$ and strongly in $C([0,2 \pi])$, which is not zero identically for $\left\|\widehat{x}_{n}\right\|_{C^{0}}=1$.

By (3.4), the sequence $g_{n}: g_{1}\left(t, x_{n}(t)\right)\left\|x_{n}(t)\right\|_{C^{0}}^{-1}$ is bounded in $L^{1}(0,2 \pi)$ uniformly in $t \in[0,2 \pi]$. Therefore, passing to subsequences if necessary, we can assume that $g_{n} \rightarrow g_{0}$ in $L^{1}(0,2 \pi)$ (for example, see [10, IV, 8.6]), and $\lambda_{n} \rightarrow \lambda$. Hence, passing to the weak limit in (3.10), we get

$$
\widehat{x}^{\prime \prime}(t)+c \widehat{x}^{\prime}(t)+\left(\lambda g_{0}(t)+(1-\lambda) \gamma(t)\right) \widehat{x}(t)=0 .
$$


Now, let $V_{0}=\{t \in[0,2 \pi] \mid \widehat{x}(t)=0\}, V_{ \pm}=\{t \in[0,2 \pi] \mid \widehat{x} \gtrless 0\}$, and define $e_{ \pm}:[0,2 \pi] \rightarrow R$ by $e_{ \pm}(t)=g_{0}(t) / \widehat{x}(t)$ for $t \in V_{ \pm}$, and

$$
e_{ \pm}(t):= \begin{cases}\Gamma(t) & \text { for } t \in[0,2 \pi] \backslash V_{+} \\ \gamma(t) & \text { for } t \in[0,2 \pi] \backslash V_{-} .\end{cases}
$$

Because $g_{1}(t, x)$ is also a Carathéodory function, we have $\left|g_{n}(t)\right| \leq\left\|x_{n}\right\|^{-1} g_{r}(t)$ $\rightarrow 0$ on $[0,2 \pi]$ as $n \rightarrow \infty$, especially on $V_{0}$. And for $g_{n}-g_{0}$ weakly in $L^{1}([0,2 \pi])$, we have

$$
0 \leq \int_{V_{0}}\left|g_{0}(t)\right| d t \leq \lim _{n \rightarrow \infty} \inf \int_{V_{0}}\left|g_{n}(t)\right| d t .
$$

Hence the sequence $\int_{V_{0}}\left|g_{n}(t)\right| d t \rightarrow \int_{V_{0}} \lim _{n \rightarrow \infty}\left|g_{n}(t)\right| d t$ by the Lebesgue dominated convergence theorem, so that $g_{0}(t)=0$ for a.e. $t \in V_{0}$, and therefore we have

$$
g_{0}(t)=e_{+}(t) \widehat{x}^{+}(t)-e_{-}(t) \widehat{x}^{-}(t) \text { for a.e. } t \in(0,2 \pi) .
$$

According to the definition of $e_{ \pm}(t)$, we can get

$$
\gamma(t) \leq e_{ \pm}(t) \leq \Gamma(t) \text { a.e. on }[0,2 \pi] .
$$

In fact, suppose that there exists a subset $U$ of $V_{+}$of positive measure such that $\gamma(t)>e_{+}(t)$ for $t \in U$. Then, by (3.16), we have

$$
\int_{U} g_{0}(t) d t=\int_{U} e_{+}(t) \widehat{x}^{+}(t) d t<\int_{U} \gamma(t) \widehat{x}^{+}(t) d t
$$

Because

$$
\begin{aligned}
\lim _{n \rightarrow \infty} \inf g_{0}(t) & =\lim _{n \rightarrow \infty} \inf g_{1}\left(t, x_{n}(t)\right)\left\|x_{n}(t)\right\|_{C^{0}}^{-1}=\lim _{n \rightarrow \infty} \inf \left(g_{1}\left(t, x_{n}(t)\right) x_{n}^{-1}(t) \widehat{x}_{n}(t)\right) \\
& \geq \lim _{x \rightarrow \infty} \inf g_{1}(t, x) x^{-1} \cdot \lim _{n \rightarrow \infty} \widehat{x}_{n}(t) \geq \gamma(t) \widehat{x}(t),
\end{aligned}
$$

using Fatou's lemma, we have that $\lim _{n \rightarrow \infty} \inf g_{n}(t)$ is integrable, and

$$
\int_{U} \gamma(t) \widehat{x}^{+}(t) d t \leq \int_{U} \lim _{n \rightarrow \infty} \inf g_{n}(t) d t=\int_{U} g_{0}(t) d t
$$

which conflicts with (3.18). Similarly we can get the other inequalities in (3.17).

Therefore, by (3.16) and (3.17), if we let $p^{+}(t)=\lambda e_{-}(t)+(1-\lambda) \gamma(t), p^{-}(t)=$ $\lambda e_{+}(t)+(1-\lambda) \gamma(t)$, then $\widehat{x}(t)$ is a $2 \pi$-periodic solution with norm $\|\widehat{x}\|_{C^{0}}=1$ of the equation

$$
\widehat{x}^{\prime \prime}(t)+c \widehat{x}^{\prime}(t)+p^{+}(t) \widehat{x}^{+}(t)-p^{-}(t) \widehat{x}^{-}(t)=0 \text { for a.e. } t \in[0,2 \pi],
$$

where $\gamma(t) \leq p^{+}(t) \leq \Gamma(t)$ and $\gamma(t) \leq p^{-}(t) \leq \Gamma(t)$.

Let

$$
p(t)= \begin{cases}p^{+}(t) & \text { for } t \in V_{+}, \\ 0 & \text { for } t \in V_{0}, \\ -p^{-}(t) & \text { for } t \in V_{-} .\end{cases}
$$

Then it is easy to check that $p(t)$ satisfies the conditions of Lemma 3. Therefore, we can conclude that (3.20) has only the trivial solution $\widehat{x}(t) \equiv 0$, which contradicts $\|\widehat{x}(t)\|_{C^{0}}=1$. This completes the proof of our theorem. 
Finally, letting $s_{0}=\max \left\{s_{1}, s_{2}\right\}$, from Lemma 2 and Theorem 1 we get

Corollary. Under the conditions of Theorem 1, there exist an $h_{0}, 0<h_{0}<1$, and an $s_{0}=s\left(h_{0}\right), s_{0}>0$, such that for all $s \geq s_{0}$ and for all $h \in C(2 \pi)$ with $\|h\|_{0} \leq h_{0}<1,(1.1)$ has at least two $2 \pi$-periodic solutions, of which one is strictly positive and the other strictly negative.

Remark. The condition (1.8) in Theorem 1 relates the asymptotic behavior of $g(t, x) x^{-1}$, for $x$ positive and sufficiently large, to the first two eigenvalues 0 and 1 of the periodic boundary value problem on $[0,2 \pi]$ for the linear operator $L=-x^{\prime \prime}$, and condition (1.7) relates the divergence behavior of $g(t, x)$ for $x$ negative and sufficiently large. On the other hand, condition (1.8) in Theorem 1 can be replaced by two other consecutive eigenvalues of periodic boundary value problem on $[0,2 \pi]$ for the linear operator $L$; for example, for some nonnegative integer $k$, the functions $\gamma(t)$ and $\Gamma(t)$ defined by (1.8) are such that $k^{2} \leq \gamma(t) \leq \Gamma(t) \leq(k+1)^{2}$, and each of the inequalities of the two extreme sides holds on a subset of $[0,2 \pi]$ of positive measure. Therefore, our corollary extends the results obtained in [1]-[3] and [5]-[7] in various directions.

\section{ACKNOWLEDGMENT}

The author wishes to thank the referee for furnishing the recently published references.

\section{REFERENCES}

[1] M.A. Del Pino, R.F. Manásevich and A.Murua, On the number of $2 \pi$-periodic solutions for $u^{\prime \prime}+g(u)=s(1+h(t))$ using the Poincaré-Birkhoff Theorem, J.Differential Equations 95(1992), $p$ p 240-258. MR 93e:34062

[2] M.A. Del Pino, R.F. Manásevich And A.Montero, T-periodic solutions for some second order differential equations with singularities, Proceedings of the Royal Society of Edinburgh 120A (1992), pp 231-243. MR 93c:34091

[3] P.DrabeK And S.Invernizzi, On the periodic BVP for the forced Duffing equation with jumping nonlinearity, Nonlinear Analysis TMA 10(1986), pp 643-650. MR 87j:34077

[4] R.E. Gaines And J.Mawhin, Coincidence degree and nonlinear differential equations, Lecture Notes in Math. 568 (Springer, Berlin, 1977). MR 58:30551

[5] J.MAWHiN AND J.R. WARD, Nonuniform non-resonance conditions at the two first eigenvalues for periodic solutions of forced Lienárd and Duffing equations, Rocky Mountain J. of Mathematics 12(1982), pp 643-654. MR 84e:34028

[6] Chengwen Wang, Generalized upper and lower solution method for the forced Duffing equation, Proc. Amer. Math. Soc. 125(1997), pp 397-406. MR 97h:34044

[7] Chengwen Wang, Multiplicity of periodic solutions for Duffing equation, Communications on Applied Nonlinear Analysis (to appear).

[8] D. Hao And S.Ma, Semi-linear Duffing equations crossing resonance points, J. Differential Equations 133(1997), pp 98-116. CMP 97:06

[9] H.Wang And Y. LI, Periodic solutions for Duffing equations, Nonlinear Analysis TMA 24(1995), pp 961-979. MR 95m:34079

[10] N.Dunford and J.T. Schwartz, Linear Operators, Part I (Insterscience Publishers, New York, 1958). MR 22:8302

Department of Mathematics and Computer Science, Rutgers University, Newark, NEW JERSEY 07102

E-mail address: chengwen@andromeda.rutgers.edu 